\title{
Special Issue: HORMESIS
}

\section{American Journal of Pharmacology and Toxicology,}

\author{
Volume 3, No. 1, 2008 \\ EDITORIAL BOARD
}

\section{Editor-in-Chief:}

Kenneth I. Maynard,

\section{Editorial Committee}

Edward Calabrese

Michael A. Dorato

Richardo Ochoa

Joseph Rodricks

\section{Corporate sponsors:}

Pfizer, (Drug Safety, Research and Development),

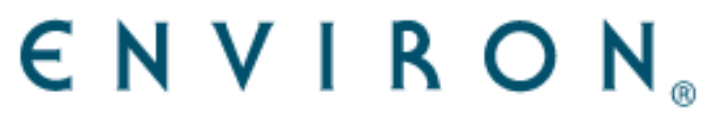

Environ International Corporation,

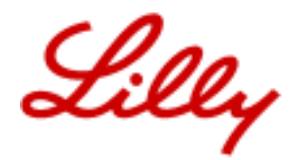

Eli Lilly and Company,

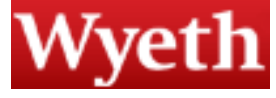

Wyeth.

Sponsored by:

science "'/" publications

www.scipub.org 


\section{Special Issue: HORMESIS}

\section{INTRODUCTION}

Hormesis is defined as "a very low dose of a chemical agent [which] may trigger from an organism the opposite response to a very high dose" (Wikipedia, the free on-line encyclopedia). It is an important dose-response phenomenon that is finally becoming recognized and integrated within the toxicological and biomedical sciences. These developments have been stimulated by the need to better understand the nature of the dose response in the low dose zone, to clarify adaptive mechanisms and how they could be exploited to enhance human health, and the need to follow up and confirm numerous investigations that have unexpectedly observed the hormetic dose-response in biomedical research, some of which have obvious clinical implications.

Only ten years ago hormesis was considered by many advocates to be a paradoxical phenomenon with questionable generalizability and by most toxicologists as a non-reproducible phenomenon born of issues with background variability, inadequate sample size and statistical power and devoid of explanatory mechanisms. This skepticism is reflected in the history of the fields of toxicology and pharmacology which failed to address the concept of hormesis in any of its major textbooks, major society conferences or academic curricula. However, much has changed in the scientific perception of hormesis and in the organization, evaluation and availability of scientific findings concerning hormesis. For example, in 1996 an hormesis database was initiated at the University of Massachusetts at Amherst based on a priori evaluative criteria which was intended to facilitate the assessment of hormetic dose responses ${ }^{[1]}$. This database has continued to be expanded and has provided a critical repository of thousands of reliable examples of hormesis, along with important information relating to its generalizability across biological models, endpoints that display hormesis and chemical classes that induce it. The database has also provided key information on the quantitative features of the hormetic dose-response, features that are important to the risk assessment process ${ }^{[2]}$ but also for more general and basic considerations such as providing insights into the quantitative features of biological plasticity within plant, microbial and animal systems.

Perhaps the most significant recent "breakthrough" occurred with two papers by Calabrese and Baldwin ${ }^{[3,4]}$ which provided a reliable estimate of the frequency of hormetic dose responses in the toxicological and pharmacological literature. These findings indicated that hormesis was observed to occur in nearly $40 \%$ of those studies which satisfied objective a priori entry and evaluative criteria. Moreover, in head-to-head comparisons with the long established threshold dose-response model, the hormesis model far our performed the threshold model in the capacity to accurately predict responses below the toxic and/or pharmacological threshold. These findings were recognized as generally important and received considerable attention in leading scientific outlets such as Nature $e^{[5]}$ and Science ${ }^{[6]}$ and numerous other scientific and popular publications (e.g. Wall Street Journal, Forbes, Fortune, US News and World Report, Boston Globe, Baltimore Sun, Scientific American, Science News, amongst others), all with prominent stories on the topic of hormesis. Such heightened interest then led to the first ever occurrence of a major workshop explicitly on hormesis at the annual meeting of the Society of Toxicology in 2006. Further supporting the perception that hormesis is gaining more scientific traction may be seen in the fact that of the nearly 1000 citations of hormesis within the Web of Science database as of mid-2008, approximately $80 \%$ of these are since 2000 . Thus, a growing body of literature across a wide range of biologic organisms, systems and the environment has heightened awareness of this scientific phenomenon of hormesis.

Despite these advances in the recognition of hormesis as an important phenomenon in the biomedical sciences, its name recognition is generally very low, and numerous other terms (e.g. U-shaped, J-shaped, biphasic, bitonic, bell-shaped, dual effects, directional effects, Arndt-Schulz Law, Hueppe's Rule, Yerkes-Dodson Law and others) have been employed which describe the same biological concept. This type of often discipline-specific terminology for the same concept is a significant problem preventing the recognition of hormesis and the centrality 
of this dose-response concept. In response, a group of nearly sixty scientists recently co-authored a paper proposing a unified dose-response terminology founded on hormesis ${ }^{[7]}$.

We think that hormesis may be a central biological principle that has been missed by the scientific and medical community until recently, with potential diverse impact from basic scientific research, drug discovery and development to governmental risk assessment. This omission is due to many interacting factors ${ }^{[8]}$ but is principally due to the fact that hormetic stimulatory responses are modest, being only about 30-60\% greater than control values, and occur below the traditional threshold. Since most experiments employ their low doses to define the threshold, hormesis would be missed in such cases. If by chance a dose entered into the hormetic zone and a modest stimulation were observed it would almost invariably be explained by "chance". Voluminous peer-reviewed research now organized in the hormesis database indicates that the much of what was considered a chance occurrence is very reproducible along with having a strong mechanistic basis. It is of importance that a potentially central concept in biology and medicine such as hormesis could have been missed by the field, thereby denying society potential benefits based on this concept.

It is our goal to further encourage the scientific evaluation of hormesis within the toxicological and pharmacological domains. To achieve this goal, some members of the editorial board of the American Journal of Pharmacology and Toxicology organized this special issue of the Journal on "Hormesis". The issue presents papers, all rigorously peer-reviewed, from diverse areas of biological and medical sciences. They cover topics including but not limited to the fields of aging, caloric restriction, cancer, cellular response, chemosensitization, drug binding, memory and synaptic plasticity, micronutrients, neuroprotection, oxidative stress and xenohormesis.

It is hoped that this set of papers will be of broad interest to biological scientists, researchers, scientific leaders, the medical and pharmaceutical community as well as governmental regulators. The aim of the editorial committee is to eliminate the marginalization of this phenomenon in science and medicine and to promote the objective evaluation, application and elucidation of the underlying mechanisms of hormesis, thus establishing its scientific foundations and biomedical implications

\section{References}

1. Calabrese, E.J. and L.A. Baldwin, 1997. The dose determines the stimulation (and poison): Development of a chemical hormesis database. Int. J. Toxicol., 16:545-559.

2. Calabrese, E.J., R. Blain, 2005. The occurrence of hormetic dose responses in the toxicological literature, the hormesis database: An overview. Toxicol. Appl. Pharmacol., 202:289-301.

3. Calabrese, E.J. and L.A. Baldwin, 2003. The hormetic dose response model is more common than the threshold model in toxicology. Tox. Sci., 71:246-250.

4. Calabrese, E.J. and L.A. Baldwin, 2001. The frequency of U-shaped dose-responses in the toxicological literature. Tox. Sci., 62:330-338.

5. Calabrese, E.J. and L.A. Baldwin, 2003. Toxicology rethinks its central belief - Hormesis demands a reappraisal of the way risks are assessed. Nature, 421(6924):691-692.

6. Kaiser, J, 2003. Sipping From a Poisoned Chalice. Science, 302:376-378.

7. Calabrese, E.J., et al. (more than 50 authors), 2007. Biological stress response terminology: Integrating the concepts of adaptive response and preconditioning stress within a hormetic dose-response framework. Toxicol. Appl. Pharmacol., 222:122-128.

8. Calabrese, E.J., 2005a. Historical blunders: How toxicology got the dose-response relationship half right. Cell Mol. Biol., 51:643-654. 